\title{
Tratamiento laparoscópico del quiste hidatídico hepático*
}

\author{
Drs. PEDRO PINTO G. ${ }^{1}$, CLAUDIO VALLEJOS O. ${ }^{1}$, EDUARDO CRUCES ${ }^{1}$, JOSÉ LOBOS G. ${ }^{2}$, \\ FRANCISCO HERNÁNDEZ G. ${ }^{1}$, MARCELO RÍOS M. ${ }^{1}$, Sr. JOSÉ GÁLVEZ M. ${ }^{3}$
}

\author{
1 Servicio de Cirugía, Hospital Regional de Coyhaique. \\ 2 Facultad de Medicina, Sede Oriente, Hospital del Salvador. \\ 3 Servicio de Pabellón, Hospital Regional de Coyhaique. \\ Chile.
}

\begin{abstract}
\section{Laparoscopic treatment of liver hydatid cysts}

Background: Laparoscopic surgery is emerging as a useful alternative for the treatment of liver hydatid cysts. Aim: To report technical data, management protocols, costs and results of laparoscopic management of liver hydatid cysts. Material and Methods: Prospective analysis of 31 patients aged 5 to 73 years (17 females), with 40 cysts, operated between 2006 and 2009. All patients received albendazol for 15 days prior to surgery and for 2 months afterwards. Surgical technique consisted in the evacuation of parasite membrane, partial excision of prominent pericystic membrane and suture of biliary communications. Results: Sixty nine percent of cysts were uni-vesicular, $74 \%$ were unique and $68 \%$ were located in the right lobe. Surgical time was $80 \mathrm{~min}$. Two patients were converted to open surgery and $24 \%$ had postoperative complications. Mean hospital stay was six days. Patients were followed for 28 months and in $4 \%$ the cyst relapsed. No patient died. The mean cost of laparoscopic treatment was US\$2.107. Conclusions: Laparoscopic technique is a useful surgical alternative for the treatment of liver hydatid cysts
\end{abstract}

Key words: Hepatic echinococcosis, laparoscopic surgery, economic cost.

\section{Resumen}

El objetivo es mostrar los detalles técnicos, protocolo de manejo, costos y resultados del tratamiento laparoscópico de quiste hidatídico hepático $(\mathrm{QHH})$. Se presenta un análisis prospectivo y descriptivo de una serie de 31 casos consecutivos de pacientes portadores de QHH operados entre enero de 2006 y enero de 2009, en el Hospital Regional de Coyhaique. Se incluye a todos los pacientes portadores de QHH tipo I, III, los tipo II y IV sintomáticos, menores a 5 cms según la clasificación de la OMS. Previo a la realización de la cirugía se les indicó 15 días de Albendazol y 2 meses en el post-operatorio. La técnica quirúrgica empleada consistió en la evacuación de la membrana parasitaria, resección parcial de la periquística prominente y sutura de las comunicaciones biliares. Siempre se utilizó drenaje. Los 31 pacientes presentaron 40 quistes, 17 de las cuales fueron mujeres, con un promedio de edad de 37 años. El estudio radiológico reveló que el 68,6 de ellos correspondió a quistes univesiculares, siendo únicos en el 74\%, localizados principalmente en el lóbulo

*Recibido el 30 de noviembre de 2010 y aceptado para publicación el 24 de enero de 2011.

Correspondencia: Dr. Pedro Pablo Pinto G. Casilla Postal 330, Coyhaique, Chile. pedropablo@patagoniachile.cl 
hepático derecho (68\%). El tiempo quirúrgico alcanzó a los 79,83 minutos. Hubo dos conversiones (6,45\%), la morbilidad alcanzó al 24\%. La estadía promedio fue de 6 días. El período de seguimiento fue de 28 meses, con una recidiva de 3,5\%. No hubo mortalidad en esta serie. El costo del tratamiento laparoscópico del QHH promedió los US 2.107. Creemos que la técnica laparoscópica, aplicada con criterio selectivo, es una alternativa útil para el tratamiento de pacientes con hidatidosis hepática, pues sus resultados son comparables a los existentes con cirugía abierta.

Palabras clave: Hidatidosis hepática, tratamiento laparoscópico, costos.

\section{Introducción}

La hidatidosis es una zoonosis parasitaria endémica en el país. Se presenta desde el extremo norte al extremo sur en todas sus regiones. Sin embargo, su distribución geográfica no es homogénea, puesto que, uno de sus huéspedes intermediarios, el ganado bovino y especialmente el ovino, es mucho más abundante en ciertas zonas de nuestro país, concentrándose en consecuencia en las zonas ganaderas ${ }^{1}$.

Las regiones más afectadas han sido clásicamente las de la Araucanía, Magallanes y Aysen, destacando nítidamente esta última con una incidencia 23 veces mayor a la nacional ${ }^{2}$.

La cirugía sigue siendo el tratamiento de elección en pacientes portadores de quistes hidatídicos hepáticos $(\mathrm{QHH})$, a pesar de su alto costo económico y a los avances experimentados tanto en el tratamiento médico como de la radiología intervencionista ${ }^{3}$.

En la última década el tratamiento laparoscópico de la hidatidosis hepática se ha vuelto más popular, experimentando un auge que ha estado en directa relación con su desarrollo tecnológico ${ }^{4}$.

Las controversias sobre el papel de la laparoscopía en el tratamiento de los QHH aún no se han resuelto, debido principalmente a la escasa experiencia mundial, falta de uniformidad en la selección de los pacientes, diversidad de técnicas quirúrgicas empleadas y ausencia de un adecuado protocolo de seguimiento ${ }^{5}$.

En esta oportunidad se desea dar a conocer un estudio prospectivo, iniciado el año 2006, sobre la utilización de la cirugía laparoscópica en el manejo de la enfermedad hidatídica hepática. El objetivo de esta comunicación es describir los detalles técnicos, protocolo de manejo, costos, revisión de la literatura y resultados.

\section{Material y Método}

Análisis prospectivo y descriptivo de una serie de 31 casos consecutivos de pacientes portadores de $\mathrm{QHH}$ intervenidos quirúrgicamente, entre enero 2006 a enero 2009 en el Hospital Regional de Coyhaique.

El protocolo de estudio utilizado incluye a todos los paciente portadores de QHH tipo I y III y los tipo II y IV sintomáticos, menores a $5 \mathrm{~cm}$, según clasificación de la $\mathrm{OMS}^{6}$. Todos fueron estudiados mediante laboratorio general y hepático, además de la realización de $\mathrm{Rx}$ de tórax y ecotomografía abdominal.

Previo a la realización de la cirugía se les indicó quince días de Albendazol (10 mg/kg/día divididos en dos dosis) y dos meses en el post operatorio, con el propósito de disminuir la posibilidad de recidiva hidatídica.

La técnica quirúrgica empleada presenta cuatro aspectos técnicos relevantes ${ }^{7}$ :

\section{Punción e inyección del escolicida}

- Previo a la aspiración del contenido del quiste se colocan gasas largas impregnadas con $\mathrm{H}_{2} \mathrm{O}_{2}$ alrededor del sitio de punción.

- Posteriormente se aspira completamente el contenido y luego se introduce el escolicida ( $\mathrm{NaCl}$ al 30\%) manteniéndose durante 3 minutos antes de volver a reaspirar.

- Se utiliza otro aspirador mientras se punciona y aspira el contenido del quiste.

- A pesar de lo anterior siempre existe el riesgo de diseminación y es por tal motivo necesario la utilización de quimioterapia profiláctica con Albendazol.

\section{Extracción de la membrana parasitaria y visualización de la cavidad quística}

- Se aspira el contenido y se extrae la membrana en una bolsa a través del trocar de $10 \mathrm{~mm}$ (Figuras 1 y 2).

- Una vez extraída la membrana se procede a revisar cuidadosamente la cavidad residual, con una óptica de $30^{\circ}$, para pesquisar y tratar eventuales comunicaciones biliares. De encontrarlas se procede a colocar puntos con Vicryl 000 (Figuras 4 y 5).

\section{Tratamiento de la cavidad residual}

Como se trata de quistes tipo I, III y II y IV menores a $5 \mathrm{~cm}$, no se consideró necesario realizar extirpación de la periquística y por ende se realizó solamente evacuación de la membrana parasitaria y resección de la periquística prominente (Figura 3). 


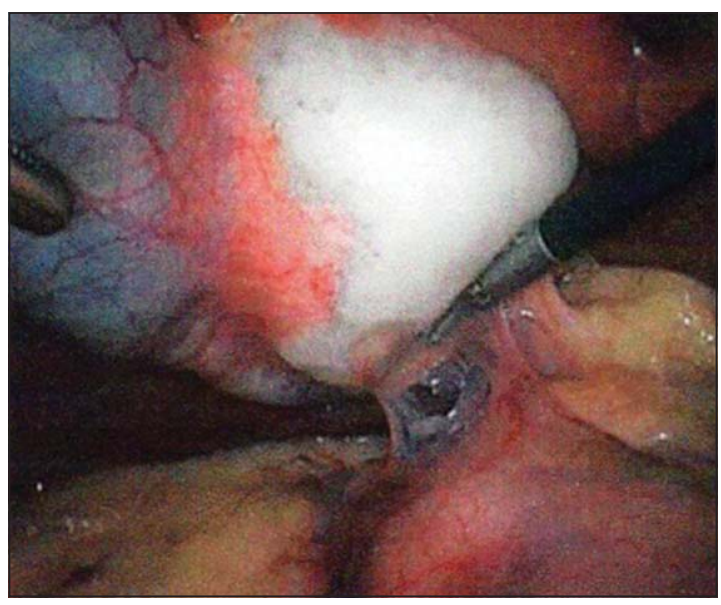

Figura 1. Quiste segmento V.

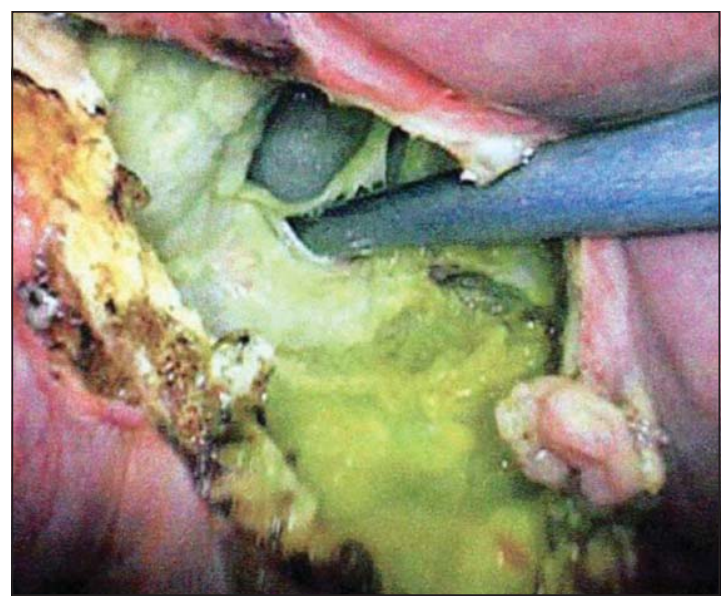

Figura 3. Resección periquística prominente.

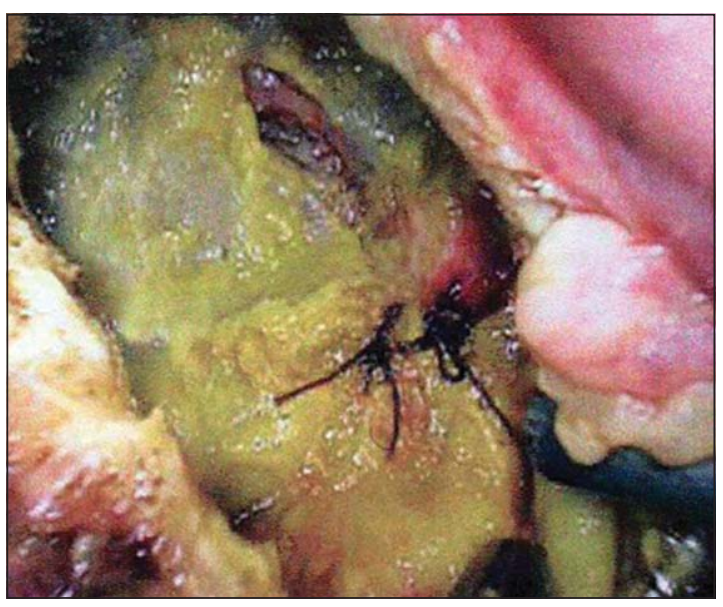

Figura 5. Sutura realizada.

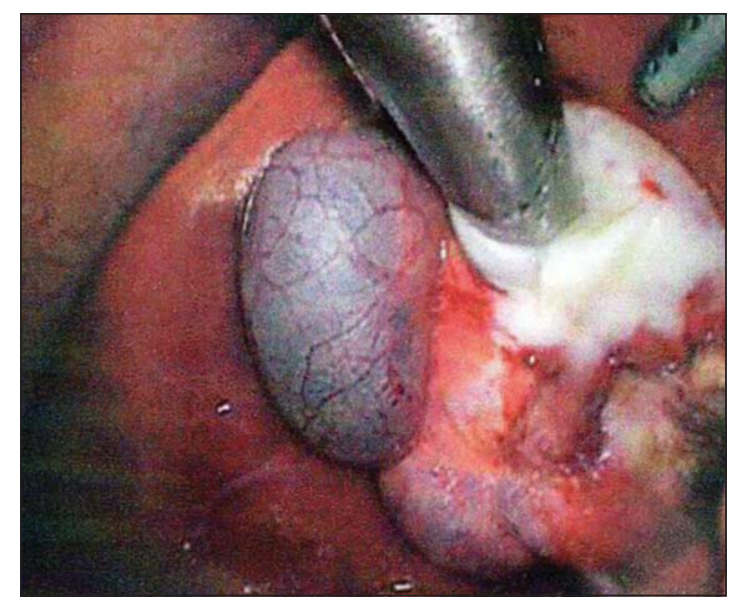

Figura 2. Aspiración de contenido.

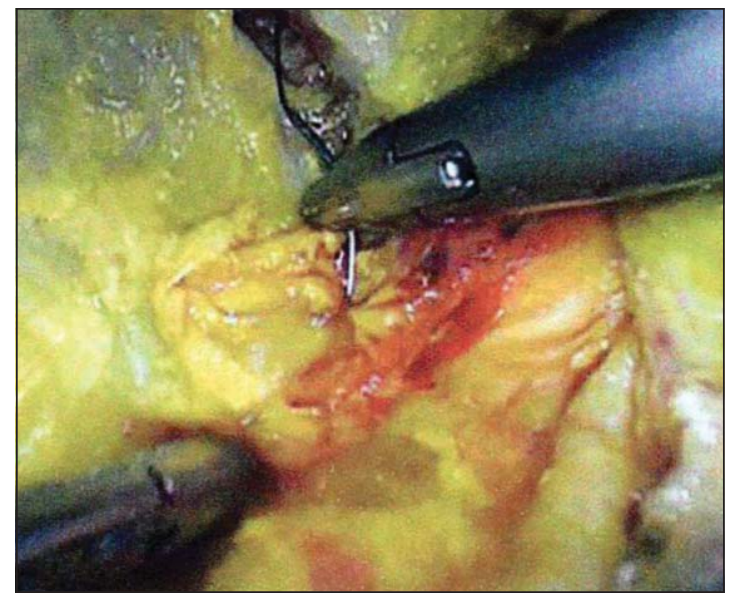

Figura 4. Sutura comunicación biliar.

\section{Uso del drenaje}

En todos los pacientes se dejó drenaje en la zona del quiste tratado, puesto que es imposible preveer la existencia o no de comunicaciones biliares ocultas.

Los controles post-operatorios se realizaron al $1^{\circ}$, $3^{\circ}$ y $6^{\circ}$ mes y luego anualmente. A todos se les practicó pruebas de laboratorio general y perfil hepático. Se dejó la realización de la ecotomografía abdominal a partir del tercero y sexto mes para luego ser solicitada anualmente. Todo lo anterior a modo de conocer la evolución y pesquisar eventuales complicaciones o recidivas.

La revisión de las fichas clínicas y tabulación de los resultados estuvo a cargo de un médico independiente al grupo de trabajo quirúrgico (J.L.G). 
Se solicitó al Jefe de Pabellón el estudio del costo quirúrgico del tratamiento laparoscópico del QHH en relación a la terapia tradicional abierta.

\section{Resultados}

Treinta y un pacientes con 40 quistes fueron considerados para el manejo laparoscópico, de ellos, 17 fueron mujeres y 14 hombres con un promedio de edad de 37 años (rango: 5-73) Tabla 1.

El estudio radiológico según clasificación OMS reveló que el 68,6\% de ellos correspondía a CE1 Tabla 2, siendo únicos en el 74\% y ubicados mayoritariamente en el lóbulo hepático derecho (68\%) Tabla 3. 160).

El tiempo quirúrgico fue de 79,83 \pm 35,5 (30-

Se debió efectuar dos conversiones (6,45\%) por presencia de bilis en la cavidad quística y el otro caso debido a la falta de visualización del quiste, la evolución fue sin complicaciones en 22 (75,86\%). La complicación más frecuente fue la fístula biliar en 3 pacientes (10,34\%), Tabla 4.

Se reoperaron dos pacientes (6,9\%) por infección de la cavidad residual, Tabla 5.

La estadía post-operatoria promedio fue de 6,06 \pm 3,02 días (1-36), siendo de 3,6 para los casos no complicados y 13,1 para aquellos que evolucionaron

Tabla 1. Características generales

\begin{tabular}{ll}
\hline Variable & Valores \\
\hline n de pacientes & 31 \\
n de quistes & 40 \\
Sexo (masculino/femenino) & $14(45,16 \%) / 17$ (58,84\%) \\
Promedio de edad (años) & $36,93 \pm 17,96$ \\
\hline
\end{tabular}

Tabla 2. Descripción ecotomográfica según clasificación OMS $2003^{6}$

\begin{tabular}{lrr}
\hline Tipo de quiste & n & \% \\
\hline a. Descritos. & 35 & 87,50 \\
CL. & 1 & 2,86 \\
CE 1. & 24 & 68,57 \\
CE 2. & 5 & 14,29 \\
CE 3. & 2 & 5,71 \\
CE 4. & 3 & 8,57 \\
b. No descritos. & 5 & 12,50 \\
\hline
\end{tabular}

con complicaciones, Tabla 6.

El promedio de seguimiento fue de 28,42 \pm 9,55 meses (16-48) habiéndose constatado hasta la fecha una recidiva $(3,45 \%)$. No hubo letalidad en esta serie, Tabla 7.

El costo de los pacientes operados de manera laparoscópica y que evolucionaron sin complicaciones alcanzó a \$1.004.962 (US: 2.107) contra \$ 975.762 (US: 2.045) de aquellos que fueron intervenidos de

Tabla 3. Anatomía patológica

\begin{tabular}{lcc}
\hline Variables & $\mathbf{n}$ & $\mathbf{\%}$ \\
\hline Quistes únicos & 23 & 74,19 \\
Quistes múltiples & 8 & 25,81 \\
Promedio diámetro mayor & $8,28 \pm 3,67 \mathrm{~cm}$ & \\
Ubicación del quiste & & \\
Lóbulo hepático derecho & 21 & 67,74 \\
Lóbulo hepático izquierdo & 4 & 12,90 \\
Ambos lóbulos hepáticos & 6 & 19,35 \\
\hline
\end{tabular}

Tabla 4. Complicaciones

\begin{tabular}{lrr}
\hline & n & \% \\
\hline 1. Complicaciones intraoperatorias & & \\
a. Sin complicaciones & 29 & 93,55 \\
$\quad$ Conversión a cirugía abierta & 2 & 6,45 \\
2. Complicaciones postoperatorias & & \\
$\quad$ (< a 30 días) & 22 & 75,86 \\
a. Sin complicaciones & 7 & 24,14 \\
b. Con complicaciones & 3 & 10,34 \\
$\quad$ Fístulas biliares & 2 & 6,90 \\
$\quad$ Infección cavidad residual & 1 & 3,45 \\
$\quad$ Colección abdominal & 1 & 3,45 \\
$\quad$ Infección sitio trabajo & & \\
\hline
\end{tabular}

Tabla 5. Reoperaciones

\begin{tabular}{lrc}
\hline Variable & n & \% \\
\hline 1. Sin necesidad de reoperación & 27 & 93,10 \\
2. Con necesidad de reoperación & 2 & 6,90 \\
Colección abdominal & 1 & 3,45 \\
Infección cavidad residual & 1 & 3,45 \\
\hline
\end{tabular}


TRATAMIENTO LAPAROSCÓPICO DEL QUISTE HIDATÍDICO HEPÁTICO

Tabla 6. Estadía hospitalaria

\begin{tabular}{lcc}
\hline Tipo de cirugía & $\begin{array}{c}\text { Promedio } \\
\text { (días) }\end{array}$ & $\begin{array}{c}\text { Rango } \\
\text { (días) }\end{array}$ \\
\hline QVL Sin complicación & $3,60 \pm 3,20$ & $1-14$ \\
QVL Con complicación & $13,12 \pm 14,06$ & $2-36$ \\
QVL general & $6,06 \pm 3,02$ & $1-36$ \\
\hline
\end{tabular}

QVL: quistectomía video laparoscópica.
Tabla 7. Seguimiento y recidiva

\begin{tabular}{lc}
\hline Variable & Valores \\
\hline Promedio seguimiento (meses) & $28,42 \pm 9,55(16-48)$ \\
Mortalidad & 0 \\
Recidiva & $1(3,45 \%)$ \\
\hline
\end{tabular}

Tabla 8. Costos de la cirugía laparoscópica y la cirugía tradicional 2010. Hospital Regional de Coyhaique

\begin{tabular}{lcccccc}
\hline Ítem & n días & $\begin{array}{c}\text { Laparoscopia } \\
\text { Costo día } \\
\text { (pesos) }\end{array}$ & $\begin{array}{c}\text { Costo total } \\
\text { (pesos) }\end{array}$ & $\begin{array}{c}\text { n días } \\
\text { Laparotomía } \\
\text { Costo día } \\
\text { (pesos) }\end{array}$ & $\begin{array}{c}\text { Costo total } \\
\text { (pesos) }\end{array}$ \\
\hline Día cama & 3 & 47.100 & 141.300 & 6 & 47.100 & 282.600 \\
Procedimiento quirúrgico & - & 336.842 & 336.842 & - & 336.842 & 336.842 \\
Derecho a pabellón & - & 127.500 & 127.500 & - & 127.500 & 127.500 \\
Anestesia & - & 130.820 & 130.820 & - & 130.820 & 130.820 \\
Medicamentos e insumos & - & 108.000 & 108.000 & - & 108.000 & 98.000 \\
Tratamiento con Albendazol & 75 & 2.140 & 160.500 & - & - & 0 \\
Costo total & & & 1.004 .962 & & & \\
Valor US (10/11/2010) & & & 2.107 & & & \\
\hline
\end{tabular}

Tabla 9. Cirugías de hidatidosis hepática en Hospital Regional de Coyhaique

\begin{tabular}{lcccr}
\hline & \multicolumn{4}{c}{ Año } \\
\hline & $\mathbf{2 0 0 6}$ & $\mathbf{2 0 0 7}$ & $\mathbf{2 0 0 8}$ & $\mathbf{2 0 0 9}$ \\
Total Cirugías (n) & 28 & 27 & 37 & 3 \\
Cirugía Tradicional (\%) & 84 & 67 & 59 & 0 \\
QVL (\%) & 16 & 33 & 41 & 100 \\
\hline
\end{tabular}

manera tradicional. Tabla 8.

Al revisar el total de pacientes portadores de QHH operados por año, durante el período estudiado, se aprecia que este alcanzó a un total de 93 pacientes, siendo resueltos por vía laparoscópica 31 de ellos, lo que equivale al 33,3\%, Tabla 9.

\section{Discusión}

Los criterios de inclusión utilizados se basaron fundamentalmente en la característica de la membrana periquística. Se privilegió su delgadez, ya que la posibilidad de que presentase comunicaciones biliares y que tal cavidad no colapsara al extraer la membrana germinativa es menor a la de aquellos quistes que presentan periquísticas gruesas ${ }^{8}$. Por tal razón se incluyeron todos los quistes tipo I y III (clasificación OMS) ${ }^{6}$ y sólo se consideró a los quistes menores de $5 \mathrm{~cm}$ de los tipo II y IV sintomáticos, puesto que los mayores de $5 \mathrm{~cm}$ presentan periquísticas fibrosas y difíciles de colapsar.

Tomando en cuenta las consideraciones antes mencionadas, hemos usado la técnica laparoscópica desde el 2006, aplicando los principios de la cirugía hidatídica hepática tradicional, que incluye la inactivación de los escólices, prevención de la filtración, eliminación de la membrana germinativa; acompañado de un adecuado manejo de la cavidad residual.

La mayor desventaja de la técnica laparoscópica es la falta de adecuadas medidas preventivas para evitar la filtración del contenido hidatídico, especialmente bajo la alta presión intra-abdominal inducida por el neumoperitoneo. Sin embargo, Bickel ${ }^{9}$, demostró que la presión intra-quística no fue mayor por el aumento de la presión intrabdominal y que el neumoperitoneo fue un elemento protector frente a una eventual filtración.

El paso más peligroso es la punción inicial y la aspiración del contenido quístico y es por tal razón que se utiliza en el pre y post-operatorio Albendazol (10 mg/kg/día) quince días en el preoperatorio y dos meses en el postoperatorio 7 . Para prevenir una even- 
tual filtración se rodea el sitio a puncionar con gasas impregnadas con $\mathrm{H}_{2} \mathrm{O}_{2}$ y se utiliza un doble mecanismo de aspiración. Nuestros pacientes no presentaron reacciones anafilácticas y en su seguimiento sólo uno de ellos presentó recurrencia (3,45\%).

En dos pacientes (6,45\%) hubo la necesidad de conversión, el primero por falta de identificación del quiste, este se encontraba en el segmento VII posterior y el otro por la presencia de una comunicación biliar que requirió de exploración de la vía biliar principal. Es indudable que con uso del ultrasonido intraoperatorio, tanto la localización como las relaciones anatómicas de los quistes serán más fáciles de detectar ${ }^{10}$.

Las técnicas laparoscópicas reportadas en el manejo del QHH se pueden dividir en dos grandes grupos, aquellos que realizan extirpación parcial o total de la periquística y las que efectúan solamente evacuación de la membrana germinativa. Es claro que las técnicas resectivas requieren de un equipamiento tecnológico y humano más avanzado. En nuestra experiencia se ha utilizado únicamente la resección de la periquística prominente, acompañada de una eventual sutura de las comunicaciones biliares.

Hasta el momento hay 16 trabajos publicados ${ }^{11-25}$ con casuísticas superiores a los 20 casos (Tabla 10), el resto de las publicaciones presenta series menores a esta cifra. El total de pacientes alcanza a los 791, y en base a estas cifras se evalúan nuestros resultados.

La morbilidad alcanzó a un 24,1\% (7 pacientes) y estuvo dada principalmente por fístulas biliares (3 pacientes) que se resolvieron mediante ERCP y complicaciones infecciosas (4 pacientes) de las cuales dos debieron ser reintervenidas para su solución definitiva.

La estadía de los pacientes que evolucionaron sin complicaciones (22 pacientes) alcanzó a 3,6 días y cuando alguno de ellos se complicó (7 pacientes), su permanencia hospitalaria casi se cuadruplicó. Cabe mencionar que como en toda nueva técnica la curva de aprendizaje es muy importante, tal es así, que en los últimos 15 pacientes no se han presentado complicaciones.

El seguimiento ha sido mayor a 16 meses en todos ellos. Es por este motivo que no se ha querido incluir nuestra casuística total, que en la actualidad

Tabla 10. Estudios publicados de tratamiento laparoscópico del QHH

\begin{tabular}{lccccccc}
\hline Autores & $\begin{array}{c}\mathbf{n} \\
\text { pacientes }\end{array}$ & $\begin{array}{c}\text { \% } \\
\text { conversión }\end{array}$ & $\begin{array}{c}\text { Estadía } \\
\text { (días) }\end{array}$ & $\begin{array}{c}\text { \% } \\
\text { morbilidad }\end{array}$ & $\begin{array}{c}\text { \% } \\
\text { mortalidad }\end{array}$ & $\begin{array}{c}\text { \% } \\
\text { recidiva }\end{array}$ & $\begin{array}{c}\text { Seguimiento } \\
\text { (meses) }\end{array}$ \\
\hline A. Alper (11) & 22 & 27 & 8 & 25 & - & 0 & $(02-17)$ \\
M. Berberoglu (12) & 87 & - & 3 & 26,4 & - & 0 & $(03-36)$ \\
G. Khoury (13) & 79 & 3 & 3 & 11 & - & 3,6 & $30(04-54)$ \\
R. Seven (14) & 30 & 23 & 6 & 22 & - & 4,3 & $17(03-72)$ \\
A. Bickel (15) & 31 & 3,3 & 6 & 19 & 3,2 & 0 & $49(09-97)$ \\
C. Manterola (16) & 8 & 0 & 2 & 0 & - & 0 & $30(23-44)$ \\
C. Kayaalp (17) & 19 & 0 & - & 0 & - & 0 & $11(03-24)$ \\
M. Ertem (4) & 48 & 4 & 4 & 6,2 & - & 0 & $34(04-78)$ \\
V. Baskaran (18) & 18 & 11 & 4 & 56 & - & 11 & $14(12-36)$ \\
K. Acarli (10) & 60 & 13 & - & 21,7 & - & 3 & $(42-132)$ \\
G. Yagci (19) & 30 & 0 & 8 & 13,3 & - & 3 & $14(08-22)$ \\
S. Georgescu (20) & 24 & 25 & 6 & 50 & - & 0 & - \\
C. Palanivelu (21) & 75 & - & - & 16,7 & - & 0 & 70 \\
W. Chen (3) & 76 & - & 7,6 & 6,5 & - & 0 & $14(06-38)$ \\
K. Maazoun (22) & 34 & - & 5 & 0 & - & 0 & $23(12-45)$ \\
E. Cassone (23) & 40 & - & 4,5 & 22,5 & - & 2,5 & 36 \\
M. Secchi (24) & 47 & - & - & 19 & - & 4,2 & $36(12-48)$ \\
H. Hason (25) & 32 & 0 & 4,3 & 9 & 0 & 0 & $16(06-25)$ \\
P. Pinto & 31 & 6,5 & 6 & 24,1 & - & 3,5 & $24(16-48)$ \\
\hline Total & 791 & $0-27$ & $2-7$ & $0-26$ & $0-3,2$ & $0-11$ & $2-132$ \\
\hline
\end{tabular}


alcanza a los 47 pacientes; ya que el control a largo plazo es de vital importancia en la evaluación de la técnica empleada. La recidiva, el aspecto más controvertido de esta técnica, se presentó en un paciente $(3,5 \%)$, cifra menor a la encontrada en la cirugía tradicional ${ }^{26}$.

Es importante mencionar que el costo de esta cirugía en el ámbito público alcanza a US 2.107 cifra relativamente similar a los US 2.045 de la cirugía tradicional (Tabla 8), lo que sumado a los beneficios de orden estético y de reincorporación laboral precoz la hace muy atractiva para pacientes portadores de QHH.

Es indudable que no todos los pacientes tienen indicación de cirugía laparoscópica. Según se aprecia en Tabla 9, solamente 1/3 de nuestros pacientes fueron resueltos mediante esta vía, pues tal como se menciona no es una técnica exenta de complicaciones (24,1\%). Los criterios de selección variarán de acuerdo a la experiencia del equipo tratante y la tecnología disponible.

Por lo tanto, la técnica laparoscópica, aplicada con criterio selectivo, es una alternativa útil para el tratamiento de pacientes con hidatidosis hepática, puesto que sus resultados son comparables a los existentes con cirugía abierta.

\section{Referencias}

1. Serra I, Araneda J, Araya C. Análisis regional de la hidatidosis humana y animal en Chile, 1989-1993. Bol Chil Parasitol. 1996;51:3-12.

2. DEIS, Minsal 2008.

3. Chen WBA, Xusheng LBA. Laparoscopic surgical techniques in patients with hepatic hydatid cyst. Am J Surg. 2007;194:243-7.

4. Ertem M, Karahasanoglu T, Yavuz N, Erguney S. Laparoscopically treated liver hydatid cyst. Arch Surg. 2002;137:1170-3.

5. Rihani H, El-Nabulsi B, Ziadat A, Al- Jarrah B. Laparoscopic approach to liver hydatid cyst, is it safe? JRMS 2005;12:69-71.

6. WHO Informal Working Group. International classification of ultrasound images in cystic echinococcosis for application in clinical and field epidemiological settings. Acta Tropica 2003;85:253-61.

7. Pinto P. Actualización en el diagnóstico y tratamiento de la hidatidosis hepática. Rev Chil Cir. 2008;60:561-6.

8. Pinto P. Periquistectomía total en el tratamiento de la hidatidosis hepática. Rev Chil Cir. 1990;42:224-7.

9. Bickel A, Daud G, Urbach D, Lefler E, Barasch EF, Eitan A. Laparoscopic approach to hydatid liver cyst. Is it logical? Physical, experimental, and practical aspects. Surg Endosc. 1998;12:1073-7.

10. Acarli K. Controversies in the laparoscopic treatment of hepatic hydatid disease. HPB 2004;6:213-21.

11. Alper A, Emre A, Hazar H, Ozden I, Bilge O. Laparoscopic surgery of hepatic hydatid disease: Initial results and early follow-up of 16 patients. World J Surg. 1995;19:725-8.

12. Berberoglu M, Taner S, Dilek O, Demir A, Sari S. Gasless vs gaseous laparoscopy in treatment of hepatic hydatid disease. Surg Endosc.1999;13:1195-8.

13. Khoury G, Abiad F, Geagea T, Nabout G, Jabbour S. Laparoscopic treatment of hydatid cyst of the liver and spleen. Surg Endosc. 2000;14:243-5.

14. Seven R, Berber E, Mercan S, Eminoglu L, Budak D. Laparoscopic treatment of hepatic hydatid cysts. Surgery 2000;128:36-40.

15. Bickel A, Loberant N, Singer-Jordan J, Goldfeld M, Daud G, Eitan A. The laparoscopic approach to abdominal hydatid cysts. Arch Surg. 2001;136:789-95.

16. Manterola C, Fernández O, Muñoz S, Vial M, Losada $\mathrm{H}$, Carrasco R, et al. Laparoscopic pericystectomy for liver hydatid cysts. Surg Endosc. 2002;16:521-4.

17. Kayaalp C. Evacuation of hydatid liver cysts using laparoscopic trocar. World J Surg. 2002;26:1324-7.

18. Baskaran V, Patnaik P. Feasibilily and safety of laparoscopic management of hydatid disease of the liver. JSLS 2004;8:359-63.

19. Yagci G, Ustunsoz B, Kaymakcioglu N, Bozlar U, Gorgulu S, Simsek A, et al. Results of surgical laparoscopic, and percutaneous treatment for hydatid disease of liver: 10 years experience with 355 patients. World J Surg. 2005; 29:1670-9.

20. Georgescu S, Dubei L, Tarcoveanu E, Bradea C, Lazescu D. Minimally invasive treatment of hepatic hydatid cysts. Romanian Journal of Gastroenterology 2005;14: 249-52.

21. Palanivelu C, Senthilkumar R, Rajan P, Senthilkumar K, Parthasarthi R, Rajapandian S. Palanivelu hydatid system for safe and efficacious laparoscopic management of hepatic hydatid disease. Surg Endosc. 2006;20:190913.

22. Maazoun K, Mekki M, Chioukh F, Sahnoun L, Ksia A, Jouini R, et al. Laparoscopic treatment of hydatid cyst of the liver in children. A report on 34 cases. J Ped Surg. 2007;42:1683-6.

23. Cassone E, Faccas J, Cassone E, Godoy C, Zambudio M, Riste F. Tratamiento laparoscópico de hidatidosis hepática y esplénica. Rev Argent Cir. 2007;93:235-46.

24. Secchi M, Pettinari R, Mercapide C, Bracco R, Castilla C, Cassone E, et al. Surgical management of liver hidatidosis: a multicentre series of 1412 patients. Liver International 2010;30:85-93.

25. Hasan H, El- Sayed O. Laparoscopic treatment of liver hydatid cyst. Journal of Medicine and Biomedical Sciences 2010;1:47-51.

26. Pinto P. Redondo J, Bravo G. Recurrencia post-operatoria de la enfermedad hidatídica. Rev Chil Cir. 1989; 41:74-7. 